\title{
Vigilancia por laboratorio de las enfermedades crónicas: una estrategia para las enfermedades metabólicas
}

\author{
Chronic disease surveillance by laboratory : a strategy for metabolic diseases
}

Antonio José Bermúdez F. ${ }^{1}$, Dora Beatriz Robayo G $^{2}$.

\section{Resumen}

Objetivo. Proponer una estrategia para la vigilancia por laboratorio de las enfermedades crónicas no transmisibles (ECNT), bajo la hipótesis de que los resultados de laboratorio de los analitos metabólicos se pueden distribuir en dos poblaciones con medias diferentes. Métodos. Estudio analítico, retrospectivo, con resultados de tamiz metabólico en 5241 recién nacidos, en los años 2014 y 2015, en cuatro hospitales, uno en Pereira, uno en Popayán, uno en Barranquilla y uno en Neiva, mediante Espectrometría de masas en Tándem, en un sistema TQD-Waters, kit Neobase ${ }^{\mathrm{TM}}$ para determinación de moléculas pequeńas en sangre. Por z-score se seleccionaron valores altos para construir la muestra anormal y los restantes datos constituyen la muestra normal. Se compararon las medias de las dos poblaciones para cada analito, con la prueba t, $(p<0.05)$. Resultados. Las 5241 muestras se analizaron para 44 moléculas de aminoácidos y acilcarnitinas de ácidos orgánicos. Para cada analito se observaron del $3 \%$ al $5 \%$ de valores altos (z-score $>3$ ). Las medias de las dos poblaciones, anormal y normal, difieren a un nivel significativo $(p<0.05)$. Discusión. Los estudios regionales con modelos planteados a nivel mundial para la vigilancia de ECNT, consideran factores socio-demográficos, somatometría y nutrición. El laboratorio clínico es una fuente de información, mediante el monitoreo de indicadores metabólicos. Se demostró que los resultados de análisis de una molécula se pueden agrupar y distribuir según una población anormal cuya media es un indicador objetivo. Conclusiones. Es posible utilizar los datos de laboratorio para monitoría de la variación de un analito, con el fin de aplicarlo para la vigilancia en salud pública de las ECNT.

Palabras clave. Vigilancia sanitaria, enfermedad crónica, metabolismo, indicadores de salud, prevención primaria

\section{Abstract}

Objective. propose a strategy for the surveillance by laboratory of chronical and not communicable diseases $(\mathrm{CNCD})$, under the hypothesis that laboratory results for metabolic analytes can be distributed in two populations with different averages. Methods. analytical and retrospective study, with results for metabolic screening for 5241 newborns, collected during the years 2014 and 2015, in four hospitals from Barranquilla, Pereira, Popayan and Neiva by Mass Tandem Spectrometry in a Triple Quadrupole (TQD) system, with the Neobase ${ }^{\mathrm{TM}}$ kit for determination of small molecules in blood. Results. 5241 samples were analyzed for 44 acylcarnitines for amino acids and organic acids. For each analyte there was 3 to $5 \%$ high values (z-score $>3$ ). The averages for both populations, normal and abnormal, differ to a

Instituto Nacional de Salud, Laboratorio Nacional de Referencia, Grupo de Genética crónicas.

1. Médico Cirujano, Magister Scientiae en Qumica, Universidad Nacional, epidemiólogo de campo FETP. Instituto Nacional de Salud, coordinador genética crónicas.

2. Bacterióloga, Magíster en ciencias biológicas, Universidad de Los Andes. Instituto Nacional de Salud, genética crónicas.

Correspondencia: Antonio José Bermúdez. Avenida Calle 26 Número 51-20, Bogotá. Telefono 2207700 ext 1264, abermudez@ins.gov.co

Fecha de recepción: Junio 27 de 2016 - Fecha de aprobación: Octubre 18 de 2016 
significant level $(\mathrm{p}<0.05)$. Discussion. Regional studies with models raised globally for the surveillance of $\mathrm{CNCD}$, consider socio-demographic, somatometric and nutrition factors. The clinical laboratory is a source of information, through the monitoring of metabolic indicators. Demonstrated that the results of analysis of a molecule can be grouped and distributed according to an abnormal population whose average is an objective indicator. Conclusions. It is possible to use data from laboratory to monitor variation of an analyte, in order to apply it to the CNCD public health surveillance.

Key words: Epidemiological Surveillance, Chronic Disease, Metabolism, Health Status Indicators, Primary Prevention

\section{Introducción}

En 2011, la Organización Mundial de la Salud (OMS) publicó el primer informe sobre la epidemia de carácter mundial de las enfermedades cardiovasculares, cáncer, diabetes y enfermedades respiratorias crónicas, junto con sus factores de riesgo y determinantes (1). El aporte de laboratorio a la vigilancia de estas enfermedades, las crónicas no transmisibles (ECNT) se contextualiza en el monitoreo de los factores de riesgo, específicamente los índices y marcadores metabólicos, y en un contexto más amplio, los determinantes genéticos. Son indicadores básicos que por sí mismos miden el riesgo, son autónomos. Sin embargo, su aplicabilidad depende de la correlación con los otros sistemas de información en salud, como la vigilancia que se desarrolla a través del Sistema Nacional de Vigilancia (SIVIGILA).

En Colombia se han adelantado estudios regionales con modelos planteados a nivel mundial para la vigilancia de ECNT. Entre estos se encuentran la Encuesta Nacional de Factores de Riesgo de Enfermedades Crónicas (ENFREC I Y II) $(2,3)$, realizada dos veces en la década de 1990, que sirve de línea base para estudios regionales (4), en los que se aplica la encuesta del estudio nacional enfocado en los diversos factores de riesgo. Con un enfoque más específico se desarrolló la Encuesta Nacional de Situación Nutricional (ENSIN, 2005) (5), que permitió conocer los factores de riesgo de enfermedades crónicas. Luego se publicó la versión 2010, con los resultados de la segunda Encuesta Nacional de la Situación Nutricional en Colombia 2010 - ENSIN (6).

La Encuesta Nacional de Demografía y Salud (ENDS) 2005, es un estudio de cobertura nacional, con representatividad tanto urbana como rural, por seis regiones, 16 subregiones y por departamentos. En esta se recopiló información sobre factores socio-demográficos y se obtuvieron medidas antropométricas, que sirvieron de insumo para los planificadores de salud, actualizados en la Encuesta Nacional de Salud (ENS) (7) versión año 2007 y la encuesta nacional de demografía y salud 2010 (8). También se ha aplicado la iniciativa CARMEN (9), que se refiere al Conjunto de Acciones para la Reducción Multifactorial de Enfermedades No transmisibles, para la región de las américas. La iniciativa de la Organización Panamericana de la Salud (OPS) tiene por finalidad promover y establecer programas y políticas globales integradas de prevención y control de las ECNT a nivel nacional y subregional en las Américas, a fin de contribuir al cumplimiento de la Estrategia Regional sobre Prevención y Control de las Enfermedades Crónicas.

La propuesta actual está contenida en el método STEPwise de vigilancia, que es un enfoque simple y estandarizado para reunir, analizar y distribuir información de ECNT en los países que son miembros de la OMS (10). 
Todos los países pueden utilizar la información de STEPS no sólo para vigilar las tendencias dentro del país, sino también para hacer comparaciones entre países. Todos los países tienen acceso a las mismas preguntas estandarizadas y protocolos de STEPS. Este método alienta la colección de pequeños y útiles pedazos de información dentro de un recurso regular y continuo. El método está focalizado en obtener datos básicos sobre los factores de riesgo que determinan las enfermedades crónicas más comunes. Cada país tiene la flexibilidad de ampliar las variables básicas y los factores de riesgo, e incorporar módulos opcionales relacionados con temas de interés local o regional. El Instrumento STEPS abarca tres niveles para la evaluación de factores de riesgo: el cuestionario, la antropometría y las medidas bioquímicas.

Este método está operacionalizado por la OMS a través de instrumentos y manuales estandarizados y validados que permiten la aplicación idéntica en cualquier región del mundo y la comparabilidad sin sesgos metodológicos (11). En Colombia se aplicó en el estudio de Factores de riesgo para enfermedades crónicas en el departamento de Santander (12), para fortalecer las intervenciones y la implementación de políticas de promoción de estilos de vida saludables. Sin embargo, no se implementó como programa continuo de vigilancia.

A pesar de los estudios mencionados, no se cuenta en el país con un protocolo establecido para la vigilancia de ECNT, ni se contempla en el Sistema de Vigilancia en Salud (SIVIGILA). Bajo este argumento, se planteó la necesidad de utilizar al laboratorio clínico como fuente primaria de información, mediante el monitoreo de indicadores metabólicos.

La vigilancia de las ECNT en los individuos de una población, mediante marcadores metabólicos a través de cohortes de seguimiento, es una estrategia costosa y con dificultades en su buen desarrollo por problemas como la movilidad de las personas y adherencia al protocolo. En su lugar, cabe la posibilidad de enfocar de manera poblacional la medición de indicadores metabólicos para la monitoria de las ECNT, principalmente con fines de seguimiento y evaluación de las medidas de intervención, para lo cual la vigilancia por laboratorio debe integrarse en sistemas nacionales de información sanitaria.

Se plantea la hipótesis de un modelo de vigilancia por laboratorio, en el cual la información delos resultados sirve como indicador de la tendencia de la ECNT, independientemente de otros aspectos dela vigilancia epidemiológica, pero con complementariedad. Esta hipótesis tiene como premisa fundamental que los resultados de la medición de una molécula cumplen una distribución normal, lo que permite identificar los valores anormales como datos fuera de la distribución, verdaderos Outlayers, que en conjunto constituyen una muestra de la población con valores altos, que es la población anormal y se puede describir estadísticamente. Si se realiza alguna intervención que cause un efecto, este se verá reflejado en los parámetros estadísticos que describen esa población anormal. Por ejemplo, en la medida en que la intervención es efectiva, se disminuye el tamaño de la muestra con valores altos, o se modifica la media con relación a la media de la población normal o de referencia.

De esta manera, la vigilancia poblacional por laboratorio se puede hacer a través del monitoreo del parámetro metabólico y evaluar su variación en el tiempo, con relación a alguna intervención o según la dinámica socio-demográfica. Para probar el modelo de vigilancia, se analizan datos provenientes del laboratorio para algunos parámetros metabólicos obtenidos en población general para identificar posibles casos de enfermedad metabólica mediante tamizaje neonatal, con el fin ulterior de proponerlo como modelo para la vigilancia de las ECNT.

\section{Métodos}

Se realizó un estudio analítico, retrospectivo, con base en los resultados del tamizaje metabólico en una muestra anonimizada de 5241 recién nacidos, realizada en los años 2014 y 2015, en cuatro 
hospitales, uno en Pereira, uno en Popayán, uno en Barranquilla y uno en Neiva, (¿ciudades?) participantes en el estudio piloto para el tamiz neonatal en Colombia. Las muestras fueron analizadas por el método de Espectrometría de masas en Tándem en un sistema TQD Waters y el kit Neobase $^{\mathrm{TM}}$ MSMS de Perkin Elmer estandarizado para la determinación de moléculas pequeñas en muestras de sangre seca.

Para cada analito se determinó su distribución normal y se calcularon los estadísticos de tendencia central. Se hizo el ajuste de la serie de datos mediante el algoritmo de Hubber (algoritmo A) (13), para identificar valores atípicos como outlayers verdaderos, ajustando con ocho iteraciones o hasta obtener estabilidad en la media y en la desviación estándar en la quinta cifra decimal. Con la muestra ajustada se calcula la estadística descriptiva de la población considerada normal y con los datos excluidos, que corresponden a un z-score superior a tres (z-score $>3$ ), se establece la población con resultados altos.

Para fines de probar la hipótesis, se seleccionaron los 10 analitos con más alto porcentaje de positividad, para lo cual se ordenaron los 5241 resultados y se determinó el porcentaje de positividad, según el número de casos con resultado de z-score superior a 3 (z-score $>3$ ).

Se realizó la comparación de las medias de las dos poblaciones, la normal y la de valores altos, mediante la prueba $t$ para comparación de medias en dos poblaciones con varianzas desiguales con un nivel de significancia de $(p<0.05)$. Se utilizó el software Open Epi de uso libre (14-19).

\section{Resultados}

Cada muestra tiene resultados para 44 analitos, de los cuales 13 son para amino-ácidos y los restantes 31 son acyl-carnitinas que se relacionan con el metabolismo de los ácidos grasos y los problemas de la $\beta$-oxidación de los ácidos grasos.
Se seleccionaron los diez analitos que presentaron más casos positivos, Tabla 1 .

Entre $172(3.42 \%)$ y $294 \quad(5.78 \%)$ casos mostraron resultados elevados, el más alto para la isovalerilcarnitina C5. Para estos diez analitos se extrajo la muestra de los valores altos (z-score >3) y se obtuvo la población con valores normales para cada analito. Una vez obtenidas la muestra normal y la muestra de valores altos, se obtuvo la estadística descriptiva para cada una, Tabla 2. Luego se compararon las medias con la prueba $t$, obteniéndose valores de $p<0.0000001$ para todos los analitos, Tabla 3.

Se construyeron los histogramas de frecuencia para la población normal y la muestra de valores altos para la molécula C5, en los que se observa la separación de las dos distribuciones, Figuras 1 y 2, y la observación conjunta pone de manifiesto que esos valores altos corresponden al percentil 0.99, Figura 3. Lo mismo se observa para la molécula fenilalanina, Figuras 4, 5 y 6. Para las demás moléculas no se muestran los resultados, pero el comportamiento es similar.

Se realizó la comparación de la serie de datos completa para cada analito sin eliminar los datos altos con la serie sin los valores altos, con el fin de evaluar si la diferencia de las medias es significativa, de tal forma que se pueda utilizar como indicador. La diferencia fue significativa para todos los analitos, pero solamente se muestra para la carnitina libre y fenilalanina, Tabla 4.

\section{Discusión}

Es claro que hay una prueba de hipótesis en los 10 casos seleccionados, con valores significativos de $p<0.0000001$ en todos los casos. Se puede afirmar que en el caso de analitos que reflejan el metabolismo, como es el caso de la isovalerilcarnitina o del amino-ácido fenilalanina, los valores altos en una población se pueden diferenciar claramente de los valores normales por medio del análisis estadístico 
Tabla 1. Analitos según porcentaje de positividad, para los diez más frecuentes.

\begin{tabular}{|cccc|}
\hline Analito & & n & \% \\
\hline NB_C5 & Isovalerilcarnitina & 289 & 5,78 \\
\hline NB_C4 & Butirilcarnitina & 284 & 5,68 \\
\hline NB_C18:2 & Linoleilcarnitina & 248 & 4,96 \\
\hline NB_SA & Succinil acetona & 220 & 4,4 \\
\hline NB_ORN & Ornitina & 218 & 4,36 \\
\hline NB_C8:1 & Octenoilcarnitina & 194 & 3,88 \\
\hline NB_PHE & Fenilalanina & 185 & 3,7 \\
\hline NB_C4DC+C5OH & Metil malonilcarnitina + hidroxiisovalerilcarnitina & 180 & 3,6 \\
\hline NB_TYR & Tirosina & 179 & 3,58 \\
\hline NB_ALA & Alanina & 171 & 3,42 \\
\hline
\end{tabular}

Tabla 2. Descripción estadística por analito, para la población normal y la muestra de valores altos agrupados.

\begin{tabular}{|c|c|c|c|c|c|c|c|c|c|c|}
\hline $\begin{array}{l}\text { Muestra de } \\
\text { valores altos } \\
\text { agrupados }\end{array}$ & C5 & C4 & C18:2 & $\begin{array}{l}\text { Succinil } \\
\text { acetona }\end{array}$ & Ornitina & C8:1 & fenilalanina & $\begin{array}{l}\text { C4DC } \\
\text { C5OH }\end{array}$ & Tirosina & Alanina \\
\hline $\mathrm{n}$ & 294 & 298 & 252 & 220 & 222 & 198 & 186 & 183 & 184 & 172 \\
\hline $\begin{array}{c}\text { media } \\
(\mu \mathrm{mol} / \mathrm{l})\end{array}$ & 0,24136 & 0,70644 & 0,56690 & 1,956 & 217,709 & 0,17308 & 98,40870 & 0,37967 & 260,173 & 579,379 \\
\hline mediana & 0,22 & 0,63 & 0,51 & 1,885 & 198,075 & 0,16 & 93,1 & 0,36 & 233,565 & 538,015 \\
\hline percentil 1 & & & 0,4 & 1,6 & & 0 & & 0 & & \\
\hline percentil 99 & 0,5035 & 1,8281 & 1,14 & & & 0 & & & & 953,806 \\
\hline SD & 0,06828 & 0,28217 & 0,17049 & 0,3473794 & & 0,03614 & 17,68074 & 0,08675 & & 113,539 \\
\hline Var & 0,00464 & 0,07935 & 0,02895 & 0,120124 & 2680,28 & 0,00130 & 310,9281 & 0,00748 & 4744,96 & 12816,2 \\
\hline $\begin{array}{l}\text { Población } \\
\text { con valores } \\
\text { normales }\end{array}$ & C5 & C4 & C18:2 & $\begin{array}{l}\text { Succinil } \\
\text { acetona }\end{array}$ & Ornitina & C8:1 & fenilalanina & $\begin{array}{l}\text { C4DC } \\
\text { C5OH }\end{array}$ & Tirosina & Alanina \\
\hline $\mathrm{n}$ & 4947 & 4943 & 4989 & 5021 & 5019 & 5043 & 5055 & 5058 & 5057 & 5069 \\
\hline $\begin{array}{c}\text { media } \\
(\mu \mathrm{mol} / \mathrm{l})\end{array}$ & 0,09607 & 0,26026 & 0,17174 & 0,8443696 & 86,5875 & 0,05898 & 47,32572 & 0,16699 & 98,3381 & 267,072 \\
\hline mediana & 0,09 & 0,25 & 0,15 & 0,76 & 81,68 & 0,06 & 45,65 & 0,16 & 92,9 & 258,41 \\
\hline percentil 1 & 0,04 & 0,1242 & 0,05 & 0,46 & 38,169 & 0,02 & 26,6324 & 0,08 & 38,7436 & 145,512 \\
\hline percentil 99 & 0,18 & 0,49 & 0,41 & 1,58 & 161,229 & 0,12 & 78,4246 & 0,29 & 191,449 & 451,610 \\
\hline SD & 0,03163 & 0,08293 & 0,08662 & 0,2789262 & 28,1112 & 0,02445 & 11,51162 & 0,04697 & 34,3468 & 69,1180 \\
\hline Var & 0,00100 & 0,00687 & 0,00750 & 0,0777843 & 790,083 & 0,00059 & 132,4911 & 0,00220 & 1179,47 & 4776,34 \\
\hline
\end{tabular}

Tabla 3. Valores de $t$ para la comparación de medias y valor de $p$ con significancia al nivel de $p<0.05$ para la población normal y la muestra de valores altos agrupados.

\begin{tabular}{|c|c|c|c|c|c|c|c|c|c|c|}
\hline \multicolumn{11}{|c|}{ Comparación de medias entre la muestra de valores altos y la población normal } \\
\hline & C5 & C4 & C18:2 & $\begin{array}{l}\text { Succinil } \\
\text { acetona }\end{array}$ & Ornitina & C8:1 & Fenilalanina & $\begin{array}{l}\text { C4DC } \\
\text { C5OH }\end{array}$ & Tirosina & Alanina \\
\hline Estadístico t & 36,1247 & 27,1396 & 36,4339 & 46,6149 & 37,2994 & 43,9567 & 38,9773 & 32,9279 & 31,5647 & 35,7188 \\
\hline $\mathrm{p}(\mathrm{T}<=\mathbf{t})$ & $\begin{array}{c}4,396 \mathrm{E}- \\
111\end{array}$ & $\begin{array}{c}1,2062 \mathrm{E}- \\
82\end{array}$ & $\begin{array}{c}1,688 \mathrm{E}- \\
103\end{array}$ & $\begin{array}{c}3,591 \mathrm{E}- \\
119\end{array}$ & $\begin{array}{c}1,511 \mathrm{E}- \\
98\end{array}$ & $\begin{array}{c}1,102 \mathrm{E}- \\
105\end{array}$ & $1,4216 \mathrm{E}-9$ & $\begin{array}{c}2,7343 \mathrm{E}- \\
79\end{array}$ & $\begin{array}{c}2,1054 \mathrm{E}- \\
76\end{array}$ & $\begin{array}{c}5,0302 \mathrm{E}- \\
82\end{array}$ \\
\hline
\end{tabular}

Tabla 4. Valores de $t$ para la comparación de medias y valor de $p$ con significancia al nivel de $p<0.05$ para la población original y la población sin valores altos

\begin{tabular}{ccc}
\hline & C0 & Fenilalanina \\
\hline Estadístico $\mathbf{t}$ & 10,1438521 & 6.8643 \\
\hline $\mathbf{p}(\mathbf{T}<=\mathbf{t})$ & $4,7362 \mathrm{E}-24$ & $3,5447 \mathrm{E}-12$ \\
\hline
\end{tabular}




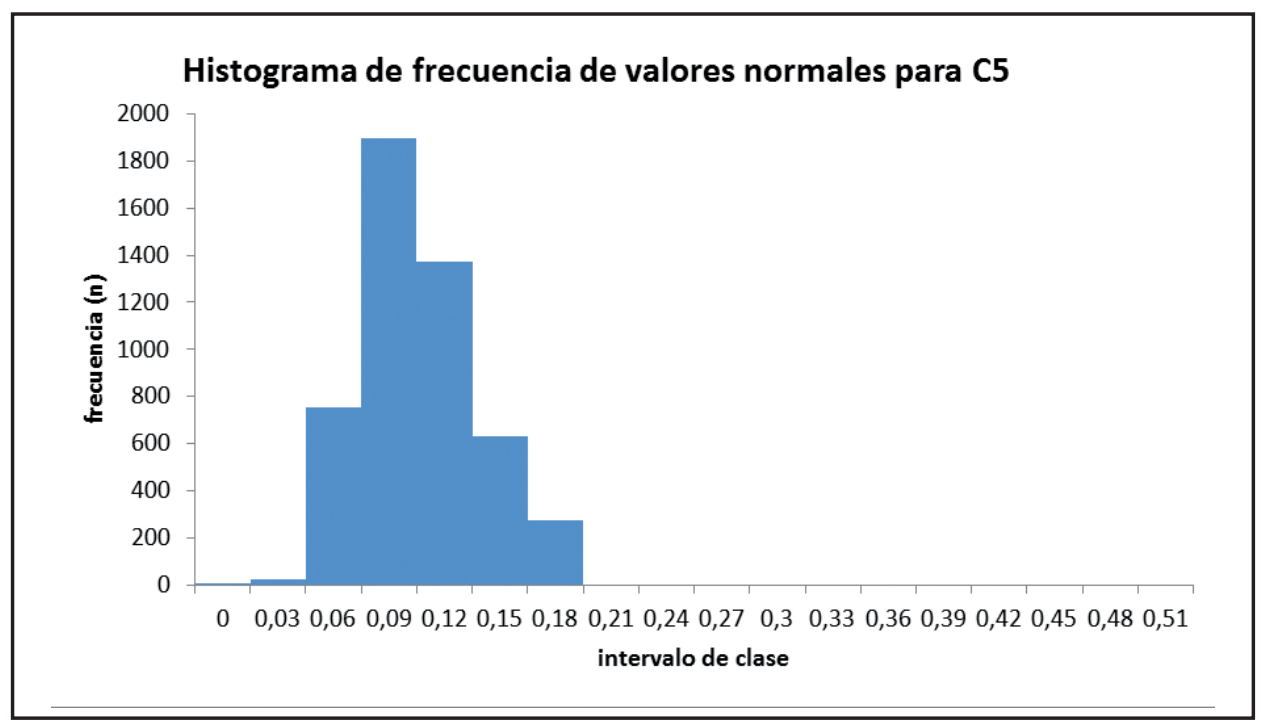

Figura 1. Histograma de frecuencia de valores normales para la molécula C5

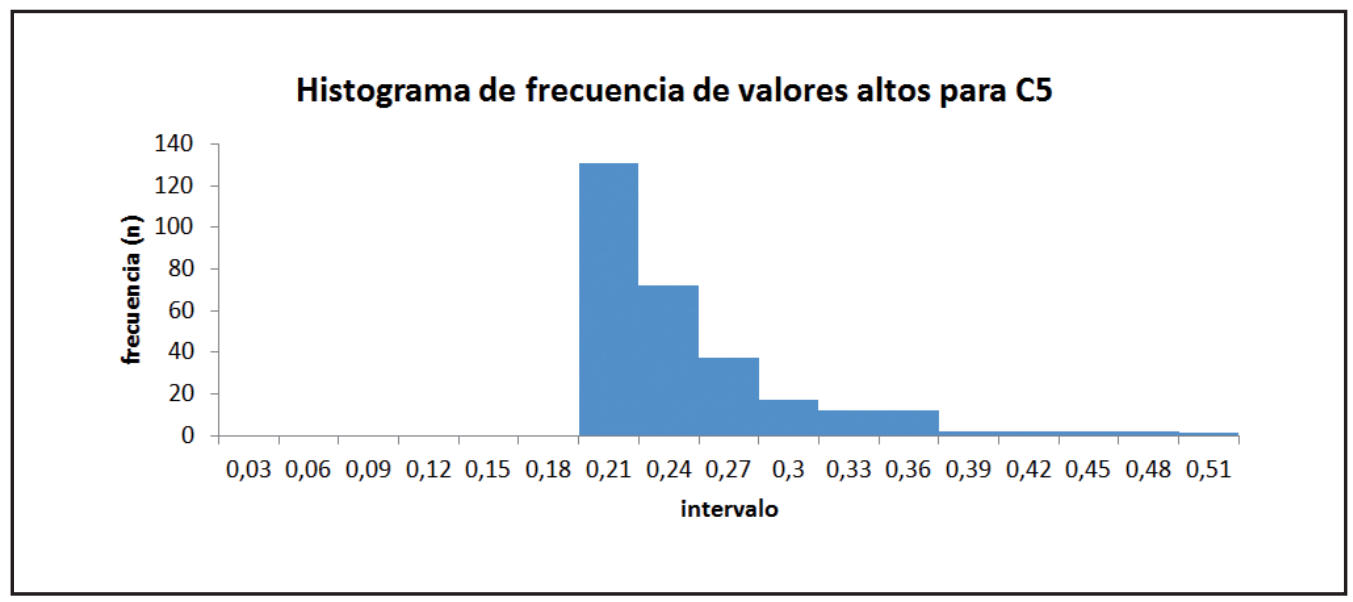

Figura 2. Histograma de frecuencia de valores altos para la molécula C5

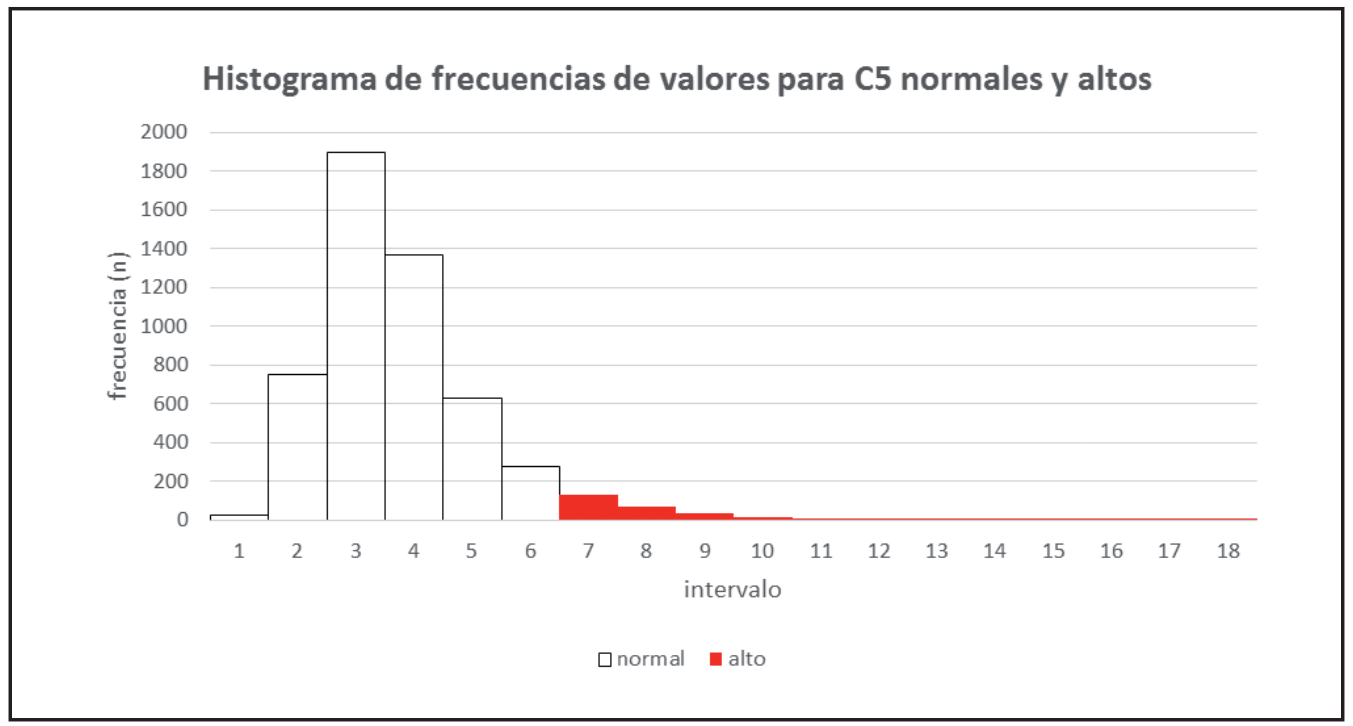

Figura 3. Distribución de frecuencia de valores normales y altos para la molécula C5 


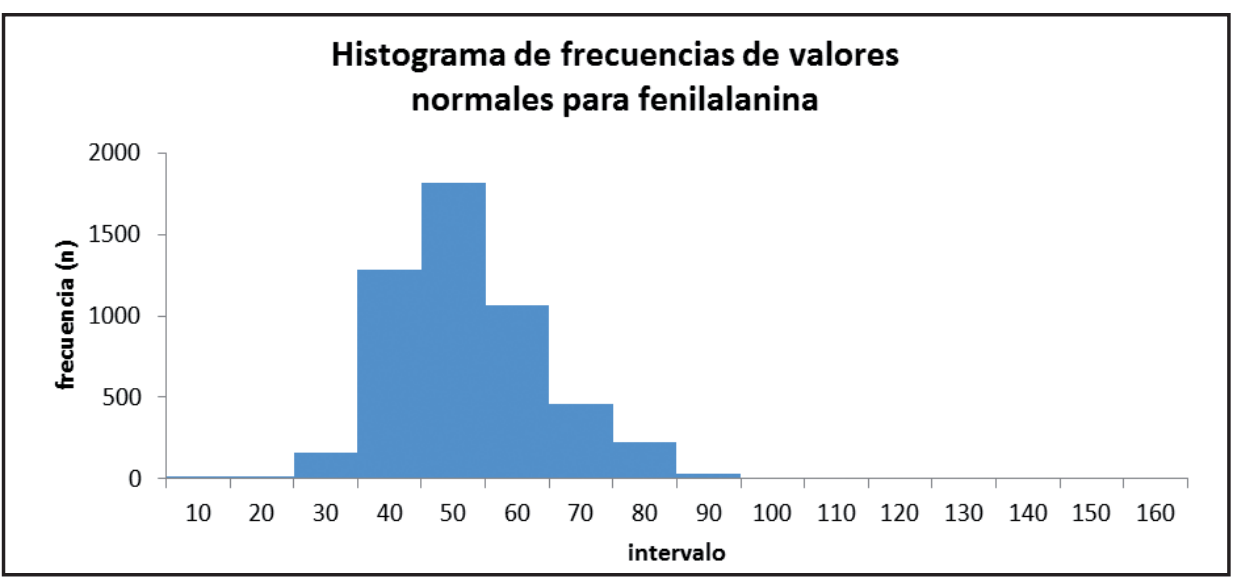

Figura 4. Histograma de frecuencia de valores normales para la molécula fenilalanina

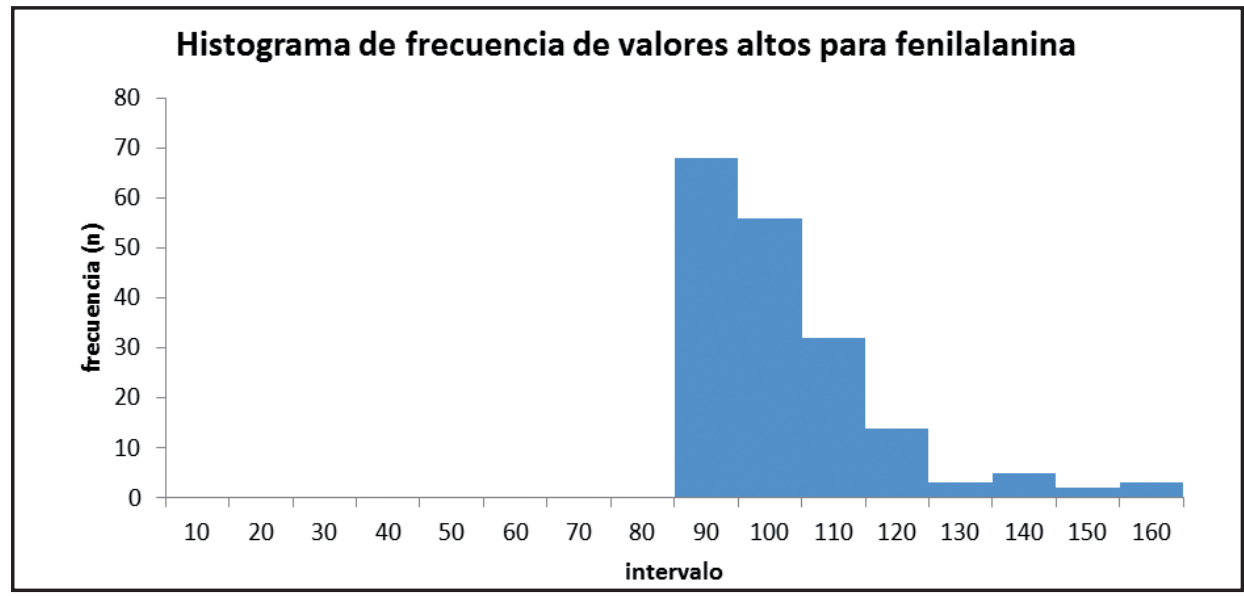

Figura 5. Histograma de frecuencia de valores altos para la molécula fenilalanina

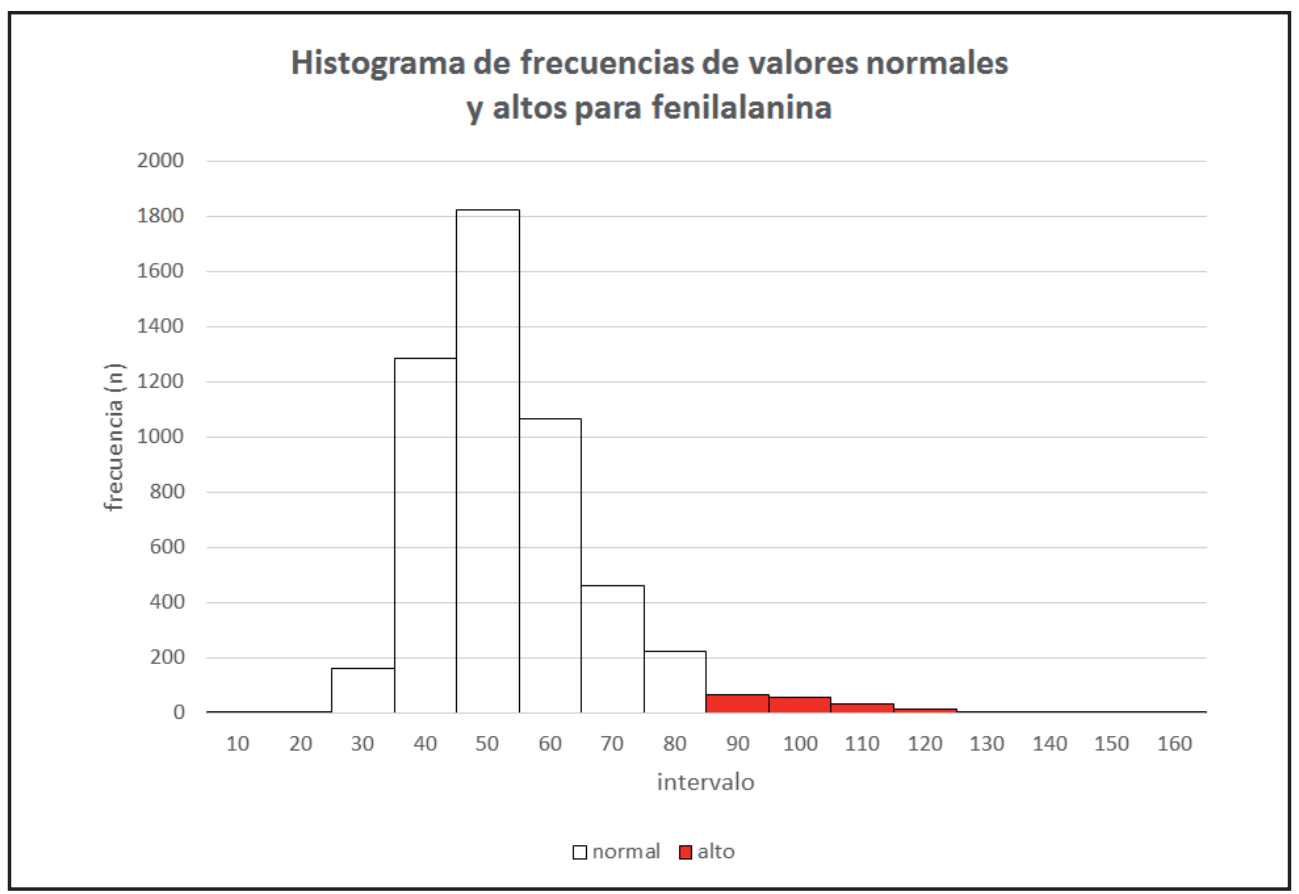

Figura 6. Distribución de frecuencia de valores normales y altos para la molécula fenilalanina 
de la media y de ahí se puede concluir que la medición de la media poblacional para valores anormales se puede desplazar si en la población se reducen los casos por alguna intervención o de manera espontánea, indistintamente.

Se puede aprovechar esta característica, para hacer el monitoreo de los parámetros metabólicos en orden a definir si los hábitos en la población están siendo modificados de alguna manera. Esto significa que se pueden tomar los datos de la población, para un metabolito cualquiera, y hacer el tratamiento estadístico para identificar los outlayers y luego caracterizar la media de esos outlayers, la cual puede ser utilizada como trazador de efecto de intervenciones. Por ejemplo, se comienza una campańa para promover el ejercicio en la población, con la esperanza de que bajen los colesteroles en ella, entonces se puede medir el impacto a través de la comparación de como varía la media poblacional de valores altos.

De esa manera, se reducen costos en la vigilancia, dado que se necesitaría mantener un monitoreo permanente de los valores de colesterol en la población, para seguir con el mismo ejemplo, sin necesidad de individualizar, dado que eso constituye un modelo de cohorte de seguimiento que es difícil de garantizar su continuidad. Con el modelo planteado, que se aproxima a un modelo de epidemiología de rebańo, desde el laboratorio, se puede garantizar un monitoreo de las ECNT de manera económica y efectiva, dado que las poblaciones siempre proveerán esta información a través de los laboratorios clínicos.

Los sesgos que se pueden presentar son, por ejemplo, que las personas que más acuden a los controles de laboratorio, son las que están bajo tratamiento, generando un sesgo de sobrestimación. Sin embargo, también es una práctica cada vez más rutinaria que los trabajadores se hagan exámenes de control o que se practiquen exámenes de medicina preventiva principalmente en el área ejecutiva y de colectividades como la milicia, que en su mayoría son personas sanas.
Entonces este sesgo se puede manejar mediante una selección adecuada de los casos considerados altos, por eso se acude al z score, que es una medida estricta, mucho más cuando se trabaja mediante el algoritmo de Hubber que garantiza la selección adecuada de los extremos. Por otro lado, se tiene la limitante de que los datos de laboratorio en una población siempre serán modificados -en alguna medida- según la técnica analítica, aunque las técnicas en la actualidad se validan, se estandarizan y solo se sacan al mercado si están debidamente aceptadas en la comunidad internacional. Sin embargo, es un hecho que hay diferentes metodologías con diferentes sensibilidades y especificidades. En el caso que se utilizó para este estudio, todas las muestras fueron evaluadas por el mismo método, pero si se hubieran utilizado diferentes métodos, entonces la varianza hubiera sido mayor, afectando de todas maneras ambas poblaciones, tanto la normal como la anormal; esto es, que el limitante se puede aceptar dado que afecta por igual a ambas poblaciones comparadas.

En ese orden de ideas es posible medir el impacto de una acción de salud pública, mediante el monitoreo de datos de laboratorio, manteniendo un monitoreo permanente de la media poblacional de sus indicadores metabólicos.

La comparación de las medias entre la serie completa de datos y la serie de datos sin los outlayers, también da una diferencia significativa $(p<0.0000001)$; por lo tanto, también es factible y plausible hacer el monitoreo por laboratorio mediante el seguimiento de la media poblacional para el analito. Sin embargo, a diferencia de la media de los valores altos, en este caso no se puede tener la caracterización de la población de los valores altos que amplía el alcance de la vigilancia por laboratorio.

\section{Conclusión}

Se demostró que es posible diferenciar los valores elevados para los analitos metabólicos, agrupándolos 
en una muestra de la población con sus características estadísticas propias, lo cual permite hacer un seguimiento de la variación en el tiempo y monitorear de esa forma el impacto de las intervenciones que se puedan realizar en la población con fines preventivos. De esta manera, se plantea como estrategia para la vigilancia por laboratorio de las ECNT, realizar el seguimiento de los analitos mediante la comparación sistemática y rutinaria de las medias poblacionales y las medias de los valores altos agrupados. Esta sería una forma sencilla y económica para medir el grado de éxito de las intervenciones públicas o privadas que se implanten para disminuir el riesgo de las ECNT.

\section{Consideraciones éticas}

El trabajo se desarrolló con datos anonimizados obtenidos en el estudio piloto para la implementación del tamizaje neonatal ampliado, por lo tanto, no hay ningún riesgo con relación al manejo de muestras ni hay relación con pacientes.

\section{Referencias}

1. Chang M. Informe sobre la situación mundial de las enfermedades no transmisibles 2010. RESUMEN DE ORIENTACIÓN. Impreso por el Servicio de Producción de Documentos de la OMS, Ginebra (Suiza). 2010. 11pp.

2. Instituto de Seguro Social, Profamilia. Encuesta sobre conocimientos, actitudes y prácticas. Consumo de Tabaco y Alcohol (ENFREC I), Tomo VI. Bogotá: Profamilia; 1994.

3. Ministerio de Salud. Tercer estudio nacional de salud bucal (ENSAB III). Segundo estudio nacional de factores de riesgo de enfermedades crónicas (ENFREC II).Tomo general. Bogotá: Ministerio de Salud; 1999.

4. Carey A. Factores de riesgo de enfermedades crónicas, Valledupar, Colombia, diciembre de 2003 (continuación). Inf Quinc Epidem Nac 2004;9(6):81-88.

5. Instituto Colombiano de Bienestar Familiar. Encuesta Nacional de Situación Nutricional. Bogotá: ICBF; 2005.

6. Instituto Colombiano de Bienestar Familiar. Encuesta Nacional de Situación Nutricional. Bogotá: ICBF; 2010.

7. Ministerio de la Protección Social, Pontificia Universidad Javeriana, Cendex, SEI. Encuesta Nacional de Salud. Fundación Cultural Javeriana de Artes Gráficas JAVEGRAF. 1ra. edición Bogotá, enero de 2009.
8. Asociación Probienestar de la Familia Colombiana. Encuesta Nacional de Demografía y Salud (ENDS 2010). Bogotá: PROFAMILIA;2010.

9. Organización Panamericana de la Salud. Documento Reunión del Comité Directivo de Conjunto de Acciones para la Reducción Multifactorial de Enfermedades No transmisibles,CARMEN. La Habana Cuba 2002.32 pp. http://www.paho.org/hq/index.php?option=com_ docman \&task=doc_view \&gid $=16166 \&$ Itemid $=270$ Consultado el:05/11/2016

10. Bonita R, de Courten M, Dwyer T, Jamrozik K, Winkelmann $\mathrm{R}$. Surveillance of risk factors for noncommunicable diseases: The WHO STEPwise approach. Summary. Geneva: WHO; 2001.

11. STEPwise. Organización Mundial de la Salud. 2014. http:// www.who.int/chp/steps/Parte1.pdf consultado el: 04/03/2016 Consultado el:05/08/2014

12. Observatorio de Salud de Santander. Secretaría de Salud Departamental de Santander. Factores de riesgo para enfermedades crónicas en Santander método STEPwise. División de Publicaciones Universidad Industrial de Santander. Bucaramanga. 2011

13. INTERNATIONAL STANDARD. ISO - 13528. First edition. Statistical methods for use in proficiency testing by interlaboratory comparisons. 2005-09-01. pp 64.

14. Dean AG, Sullivan KM, Soe MM. OpenEpi: Open Source Epidemiologic Statistics for Public Health, Versión. www. OpenEpi.com, actualizado 2015/05/04; acceso 2016/03/04.

15. Nausa JG. Evaluación Clínica y radiográfica de injertos biocerámicos tipo Hidroxiapatita como alternativa en la reconstrucción de alveolos dentarios postexodoncia. Nova. 2014;12(22).

16. Ramírez LCC, Leal LCS, Rodríguez FAE. Determinación de la presencia de bacterias patógenas para el humano en aguas de riego en la cuenca alta de la sabana de Bogotá; DC Colombia. Nova. 2014;12(22).

17. Páez LCC, Quintero IDP, Suarez MCU, Mora RMS, Jiménez MG, Cano LAM. Comparación del cultivo celular de HeLa y HEp-2: Perspectivas de estudios con Chlamydia trachomatis. Nova. 2015;13(23).

18. Campuzano S, Mejía Flórez D, Madero Ibarra C, Pabón Sánchez P. Determinación de la calidad microbiológica y sanitaria de alimentos preparados vendidos en la vía pública de la ciudad de Bogotá DC. Nova. 2015;13(23):81-92.

19. Millan J, Yunda L. An Open-Access Web-based medical image atlas for collaborative Medical image sharing, processing, Web Semantic searching and analysis with uses in medical training, research and second opinion of cases. Nova. 2014;12(22):14350 